\title{
ID LIKE TO HAVE A MACHINE...
}

\section{by Orrin Frink}

It is not hard to find technical literature describing language learning equipment that already exists and advice on how to use it. Truly we live in a technical age. My paper describes two pieces of equipmer.t I would like to have, and which I have not found anywhere. They are probably not difficult for a technician to construct, although they are beyond my capabilities as an apartment dweller and professor of Russian. Still, if they are within the realm of a small departmental budget, I would like to have them so I can try them out myself in class. I would make only one general comment before getting on with describing these two pieces of equipment: that in contrast to the elements of an ordinary modern educational language laboratory, which usually is somewhat more than an extended, unified, multicomponential tape recorder-player, these two pieces are designed primarily as tools for individual and separately directed use by the teacher as diagnostic and clinical, or pedagogic-cybernetic instruments. As a teacher, I think I would like to have the capabilities that these two theoretical pieces of equipment are designed to provide. And so to the business.

\section{Design for a Tri-Modal Cassette Tape Listener-Recorder-Player.}

I'd like to have at my disposal a small, portable cassette tape recorder-player with a ten or twenty-second time delay buffer storage built into the microphone input circuitry. I have in mind a piece of equipment about the size and weight of a Sony-110 player, or volume seven of the Encyclopedia Britannica, although not necessarily the same shape. A comfortable carrying handle is important, and the equipment should provide the option of either standard plug-in $A C$ house current or self-contained battery DC poweration. It will cost under a hundred dollars so that a teacher can afford to buy one.

Using a ten-second (or twenty, if you prefer) continuous long cassette as a time-delay buffer storage, for holding, and then transferring, audio input to a secondary cassette would give the operator ten seconds to identify something he wants to record, without recording everything prior or necessitating a lengthy rewind to locate a particular spot, and without losing the initial part of the sequence to be recorded. For language learning and phonetic practice, most things said are not worth recording permanently, but one usually does not recognize something as record-worthy until it is half gone, or rather, by the time one has a chance to find and push the record button the utterance is well under way or finished. Conversely, simply recording everything said and later trying to edit out all but what is of value or 
interest to the immediate situation removes the immediacy from any feedback possibility, and is extremely wasteful of recording media and time.

I can see myself using such a recorder in this way. I turn on my recorder and push the "listen-mode" button. In this mode, the recorder begins continuously recording on the continuous loop cassette, in effect, retaining in continuous storage the last ten seconds of audio input. I begin a conversation or pattern drill with one or more students. When I notice a student response that I wish to bring to his attention for reinforcement, correction or modification, I push the "record-mode" button on the machine within ten seconds after his beginning of the utterance. In this mode the recorder begins re-recording onto the second cassette what it finds at the end of the still running and still recording initial buffer storage cassette. Ten seconds after the student has stopped saying whatever I wanted to preserve, I can either stop the machine, rewind and play the secondary cassette back to him, to demonstrate what he has said, or I can put the recorder back into the "listen-mode" and continue listening until the next record-worthy utterance appears. Feedback and editing can be immediate. At this point I can rewind and start over again at the beginning of the secondary cassette, erasing what was just recorded, or I can continue without rewinding, from the end of the first permanent recording preserving it for later, subsequent comparison.

I predict that such a tri-modal cassette listener-recorder-player would be an extremely powerful tool in some other situations. For example, in recording a departmental meeting it would be possible to pre-edit on the spot, with an aim not so much to preserving (in the recorder "recording-mode") what seems important in immediate retrospect, but rather omitting (in the recorder "listening-mode") conversations, comments and arguments which are considered to be nonrecord-worthy in immediate retrospect. Of course, active use of the recorder described here does not preclude continuous concurrent use of a normal, long playing recorder to make a total record of the entire session of a class. As a pre-editing device for recording deep psychotherapy or as a self-confrontation device in active-directive psychotherapy, the tri-modal machine might be of some diagnostic and clinical help. In directed choral or instrumental music practice the device might be of value too, as it might in the field of public speaking, inter-personal communication or forensics. In general, I think I would like to have at my disposal the capabilities this theoretical machine would provide. 


\section{Machine}

\section{Design for a Quadri-Modal Cueing Device}

I'd like to have at my disposal a small, portable poly-modal timing device, or cueing machine about the size of a table telephone and the same color: a black box that operates on self-contained batteries or can be plugged into house current with a small light on the front, a pleasant "beeper" inside, a button on the top to start the cycle, four switches on the back, one to turn it off and on, one to place it into either the "light-mode" or the "beep-mode", one to set it to either "manual cycle" or "automatic recycle", plus a switch that pre-sets the cycle length from one to twenty seconds either continuously variable or in increments of one second.

I would use this cueing device to set the pace, speed up, slow down or control classroom language activities of the conversation, pattern drill or dictation variety. Probably fierce in appearance and hatefully persistent in operation, the device should be gentle in function, nearly silent mechanically, with the visual cue on the front about the size and intensity of a flashlight bulb, and the audio cue neither a crash, clang, rrrring, buzz or braaaack, but a pleasant "beep" of short duration sufficiently loud for classroom use, but quieter than a battery operated bicycle horm.

When set to the "automatic recycle mode", the device will produce a "beep" or flash of light at the end of however many seconds you have set the cycle-length switch to. It will begin the first cycle when you touch the button on the top, and not before, and will continue producing a "beep" or flash of light every however many seconds it was until you take it out of the "automatic recycle mode".

When set to the "manual cycle mode" it will begin to count off however many seconds you have set the cycle length switch to, and produce a "beep" or flash of light at the end of that cycle, and will not initiate the next cycle until the button on the top is touched. Upon completing the current cycle, it will reset and wait for the next touch on the button. If the button is touched during execution of a cycle, it will instantly reset without producing the "beep" or flash of light, and will wait for the next push of the button.

\section{Comment}

I would like to note the practical results of publishing an earlier article, "A Design for an Audio Electronic Repeater", in the February 1964 issue of The Modern Language Journal (Vol. XIVIII, pp. 78.82.). Within two months, I received a post card from a high school student in California telling me that he was going to build the machine as his high school science project. I wrote encouragement and offered help. None was asked. In the summer he sent another postcard to say that the machine was done, and worked, and that I could come out and see 
it. To date, I have had absolutely no response to the article from anyone, written or oral, save the two post cards from California. Where are you, Electronic Industry?

As for the tri-modal cassette listener-recorder-player described above, my guess is that the mechanism and wiring are not extremely complicated, and that the basic principles already exist in the Canon "Repeat-Corder-L" which has been advertised in the back section of this journal in the October 1970, December 1970 and October 1971 issues. The machine described there is remarkably similar in function to the parameters outlined in my February 1963 article in The Modern Language Journal, (Vol. XLVIII, pp. 78-82.).

I am serious in my desire to have at my disposal both of the teaching learning aids described here, and I would be pleased to have a reaction from my colleagues who are actively involved in the teaching process.

ABOUT THE AUTHOR:

Orrin Frink is a Professor of Russian at Ohio University, Athens, Ohio.

\section{NATIONAL ASSOCIATION OF LANGUAGE LABORATORY DIRECTORS NALLD JOURNAL}

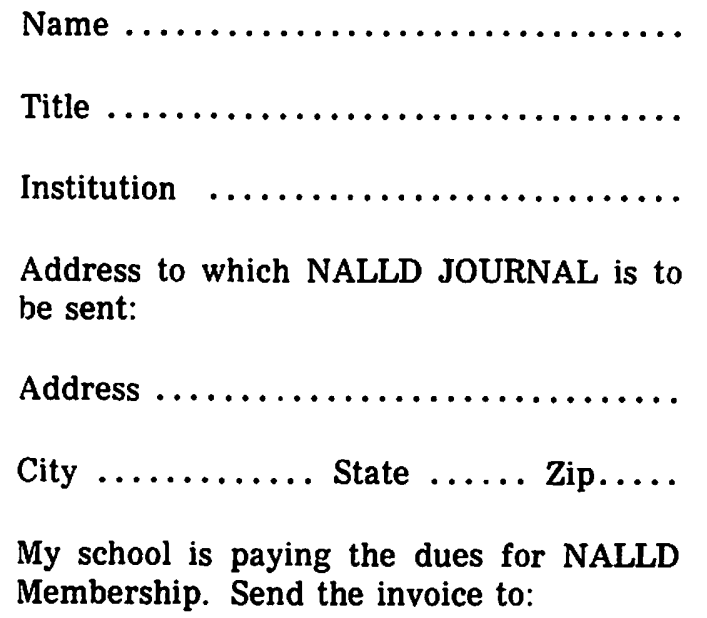

MEMBERSHIP APPLICATION

$\square$ New Member $\quad \square$ Renewal

U.S.A. (50 states)

Canada

Foreign Air Mail

Student (non-voting)

Library (non-voting)

$\$ 6.00$

$\$ 7.00$

$\$ 9.00$

$\$ 4.00$

$\$ 9.00$

Return this form to:

Mr. Dale Lally

Treasurer, NALLD

Language Laboratory

Marquette University

Milwaukee, Wisconsin 53233

Commercial organizations are invited to contact NALLD regarding associate membership.

Please mention NALLD Journal when writing advertisers 